medgen $2017 \cdot 29: 225-233$

DOI 10.1007/s11825-017-0132-8

Online publiziert: 22. Juni 2017

(c) Der/die Autor(en) 2017. Dieser Artikel ist eine Open-Access-Publikation.

CrossMark

Marcus Karlstetter · Katharina Dannhausen · Thomas Langmann

Lehrstuhl für Experimentelle Immunologie des Auges, Zentrum für Augenheilkunde, Uniklinik Köln, Köln, Deutschland

\title{
Mikroglia und Immuntherapien bei degenerativen Netzhauterkrankungen
}

Retina offensichtlich, wobei regional vor allem der Bereich um die Fovea von einer hohen Mikrogliadichte gekennzeichnet ist $[14,53]$. Dementsprechend scheint die Region um die Makula herum besonders empfänglich für zelluläre Immunaktivierung zu sein, was von hoher Bedeutung für makuläre Netzhauterkrankungen ist.

Homöostatische Mikrogliafunktionen in der unreifen Netzhaut sind mit der Kontrolle der entwicklungsabhängigen Apoptose, der Phagozytose von Zelltrümmern, der Bildung von neuronalen Verbindungen und der Orientierung des primären Netzhautgefäßwachstums verbunden $[56,59]$. Eine weitere entscheidende Funktion von Mikrogliazellen ist die Modulation der Synaptogenese. Im Gehirn führt der genetische Knockout der mikrogliaspezifischen Proteine DAP12 und CX3CR1 in der Maus zu schweren Defekten in der Synaptogenese [52]. In der unreifen Retina wird das synaptische Pruning zur korrekten Verschaltung neuronaler Netzwerk ebenfalls durch mikrogliale Phagozytose gesteuert [9]. Dabei sezernieren retinale Ganglienzellen der sich entwickelnden Netzhaut den Komplementfaktor C1q, der als Schlüsselfaktor der klassischen Komplementkaskade wirkt. Anschließend dekoriert der aktivierte Komplementfaktor C3 entsprechende Synapsen, um ihre komplementrezeptorabhängige Phagozytose in Mikroglia anzuschalten [9].

In der reifen Netzhaut bilden verzweigte Mikrogliazellen ein komplex organisiertes territoriales Netzwerk, in dem ihre Ausläufer ständig die Oberfläche der Neuronen in einem defi- nierten Bereich abscannen (• Abb. 3a). Durch ihr dynamisches Verhalten ist die Mikrogliapopulation in der Lage, in kürzester Zeit eine umfassende Überwachung der gesamten Retina zu erreichen [13]. Mikrogliazellen überwachen ihre Umgebung mit einem Repertoire von Oberflächenproteinen für Zytokine, Chemokine, Komplementkomponenten, Antikörper und besonderen Rezeptoren für veränderte Zelloberflächenstrukturen. Die hohe Empfindlichkeit dieser Mikrogliasensoren verlangt dementsprechend, dass ihre Aktivierung durch inhibitorische Mechanismen in der gesunden Retina streng kontrolliert wird. Einerseits spielen lösliche Faktoren des retinalen Pigmentepithels wie TGFbeta eine Rolle, die einen eher antiinflammatorischen Immunzellphänotyp induzieren [51]. Andererseits ist eine direkte physikalische Wechselwirkung von Mikroglia mit anderen Netzhautzellen bedeutend. Das TransmembranGlykoprotein CD200 wird auf mehreren retinalen Zellen exprimiert, einschließlich des vaskulären Endothels, der Photorezeptoren und der Ganglienzellen, und dient als inhibitorischer Ligand für den auf Mikrogliazellen exprimierten CD200-Rezeptor [49]. CD200Bindung an CD200-R auf Mikroglia löst eine inhibitorische intrazelluläre Signalkaskade aus, um die überschießende proinflammatorische Aktivierung zu blockieren [27]. CX3CL1 (Fraktalkin) ist ein weiterer Mikrogliaregulator, der konstitutiv von gesunden retinalen Neuronen und Endothelzellen freigesetzt wird und an CX3CR1 auf Mikroglia bindet, um deren Neurotoxizität zu verten ist besonders in der menschlichen 

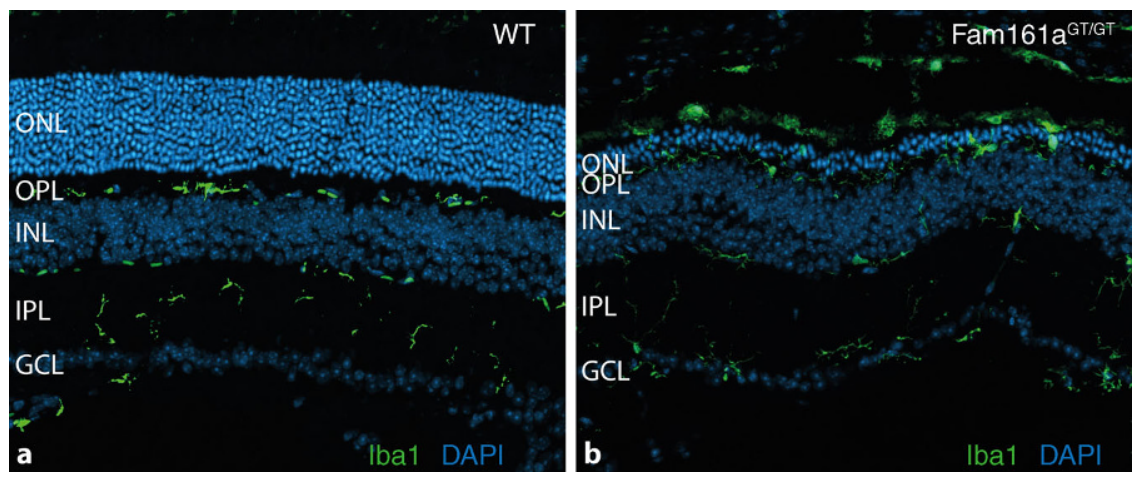

Abb. 1 ॥ Iba1-Immunfärbung (grün) von Mikrogliazellen in histologischen Schnitten einer a wildtypischen (WT) bzw. b Fam161a-defizienten Mausnetzhaut. Zur Visualisierung der Netzhautschichten wurde mittels DAPI eine Gegenfärbung der Zellkerne (blau) durchgeführt. a Ramifizierte Mikrogliazellen der gesunden Netzhaut sind in der inneren (IPL) und äußeren (OPL) plexiformen Schicht zu finden. b Durch die Degeneration der äußeren Netzhaut wird die äußere Körnerschicht (ONL) signifikant dünner und Mikrogliazellen wandern in die äußere Retina bzw. in den subretinalen Raum. (GCL ganglion cell layer)

hindern [10]. Schließlich sezernieren Müller-Zellen den diazepambindenden Inhibitor (DBI), einen Liganden für das Translokatorprotein (18 kDa) (TSPO), das in aktivierten Mikrogliazellen exprimiert wird, und begrenzen somit die Mikrogliareaktivität [32, 61].

\section{Mikroglia und Netzhaut- erkrankungen}

Die Mikrogliaaktivierung galt lange Zeit als nebensächliche Begleiterscheinung bei degenerativen Netzhauterkrankungen. Mittlerweile ist dieser Prozess jedoch als pathophysiologisch relevanter Krankheitsmechanismus anerkannt (Schaubild - Abb. 2). Die morphologische Transformation und Migration der residenten Mikroglia markiert die Aktivierungsphase dieser Reaktion. In der Effektorphase sammeln sich Mikroglia in den geschädigten Schichten an und interagieren mit infiltrierenden Blutzellen, die durch eine geschwächte Blut-Retina-Schranke eindringen können.

\section{Mikroglia und erbliche Netzhautdystrophien}

\section{Mikroglia bei Retinitis pigmentosa}

Hereditäre Netzhautdegenerationen bilden eine große Gruppe von Augenerkrankungen mit Mutationen in mehr als 250 bisher identifizierten kausalen Genen (https://sph.uth.edu/retnet/). Apop- tose und retinaler Zelltod sind wesentliche Bestandteile degenerativer Prozesse, die zusammen mit dem genetisch bedingten Funktionsverlust die strukturelle Integrität der Netzhaut schädigen [8]. Das selektive Absterben von Stäbchenphotorezeptoren bei Retinitis pigmentosa (RP), der häufigsten Form von erblichen Netzhautdystrophien, ist eng mit der Anlockung reaktiver Mikroglia assoziiert [25]. Dabei weisen die Lokalisation von Mikroglia in der äußeren Körnerschicht und große Menge phagozytierter Photorezeptoraußensegmente darauf hin, dass das sekundäre Absterben von Zapfenphotorezeptoren bei der RP möglicherweise aktiv durch Mikroglia induziert sein könnte.

\section{Mikroglia bei Mausmodellen erblicher Netzhautdystrophien}

Die erste stammspezifische Netzhautdegeneration bei Mäusen wurde vor über 90 Jahren identifiziert [36]. Diese bestens untersuchte natürliche Mauslinie, die auch als rd1 (retinal degeneration 1) bezeichnet wird, trägt eine Nonsensemutation im Pde6b-Gen, das für eine membrangebundene cGMP-Phosphodiesterase kodiert, die in der Phototransduktionskaskade in Stäbchen essenziell ist. Rd1-Mäuse zeigen einen schnellen Beginn und eine starke Netzhautdegeneration mit einem gut definierten Zeitverlauf[11]. Umfassende Genexpressionsanalysen mit Netzhautgewebe von sehr jungen rd1 Mäusen zeigten eine Reduktion der photorezeptorspezifischen Genexpression, gefolgt von erhöhten apoptose- und neuroinflammationsspezifischen Transkripten einschließlich der Überexpression des Komplementfaktors C1qa am postnatalen Tag 14 (PN14) [57]. Rd1 Netzhäute weisen zum selben Zeitpunkt eine signifikante Anzahl von proliferierenden reaktiven Mikrogliazellen in der äußeren Körnerschicht auf, die Tumornekrosefaktor und das Chemokin CCL2 sezernieren. Solche Mikrogliazellen sind auch in homozygoten rd10 Mäusen nachweisbar, die eine Missensemutation im Pde6b-Gen tragen [66]. Die Kreuzung von rd10Mäusen mit Tieren, die im CCL2-Rezeptor (Ccr2-/-) defizient sind, führte zu einer signifikanten Reduktion reaktiver Mikroglia und einer verzögerten Degeneration mit länger erhaltener ERGFunktion [24]. Dies deutet darauf hin, dass eine Hochregulierung der CCL2CCR2-Achse während der Anfangsphase der Netzhautdegeneration notwendig ist, um eine Mikrogliaaktivierung und möglicherweise Makrophagenrekrutierung auszulösen und dadurch zusätzlich Netzhautschäden zu verursachen. Unsere Arbeitsgruppe konnte die zeitliche Verbindung der Mikrogliaaktivierung mit der Photorezeptorapoptose bei Retinoschisin-defizienten (Rslh-/Y) Mäusen untersuchen, einem Modell für die X-gebundene Retinoschisis [65]. Dabei konnten DNA-Mikroarrays zeigen, dass die transkriptionelle Reaktion von Mikroglia dem Zelltod in der Netzhaut zeitlich deutlich vorausgeht [21]. Die frühe Mikrogliose steht auch in direktem Zusammenhang mit der Netzhautdegeneration im Fam161-defizienten Mausmodell der Retinitis pigmentosa [33, 42]. Mit dem Verlust der Photorezeptorschicht wandern Mikrogliazellen in die äußere Netzhaut ein ( $\bullet$ Abb. 1b) und es erfolgt die Auflösung des ramifizierten Zellnetzwerks, verbunden mit einer morphologischen Transformation zu großen neurotoxischen Phagozyten (- Abb. 3b). 


\section{Mikroglia und altersabhängige Makuladegeneration}

Die altersbedingte Makuladegeneration (AMD) ist eine multifaktorielle, komplex genetische degenerative Erkrankung der zentralen Netzhaut, die in der Spätphase zu signifikantem Sehverlust führt. In der frühen und intermediären asymptomatischen Phase zeigen sich im Fundus von Patienten Pigmentveränderungen und das Auftreten von weichen Drusen. Spätformen der AMD sind durch geographische Atrophie (trockene AMD) bzw. neovaskuläre Prozesse (feuchte AMD) gekennzeichnet. Bei der feuchten AMD kann vor allem das Einsprossen von Aderhautgefäßen im Bereich der Makula in den subretinalen Raum mit Ödembildung, Blutungen und RPEAblösungen schwere Komplikationen verursachen [30].

\section{Mikroglia bei der AMD}

Umfangreiche genomweite Assoziationsstudien und Metaanalysen belegen eine genetisch bedingte Dysregulation des Komplementsystems bei der AMD [19]. Darüber hinaus sind aktivierte Mikroglia eng mit Drusen von Patienten mit früher AMD assoziiert [37]. Patienten mit geographischer Atrophie zeigen besonders amöboide Mikroglia in der äußeren Körnerschicht [25]. Ein systembiologischer Ansatz, der die Transkriptome von menschlichen Spenderaugen mit und ohne AMD verglichen hat, zeigte eine starke Überexpression von Mikrogliarezeptoren und verschiedenen Komplement- und Chemokin-mRNAs in AMDProben. Dies deutet darauf hin, dass die Mikrogliaaktivierung zu den frühzeitig auftretenden und lang anhaltenden chronischen Mechanismen bei der AMDPathogenese zählt [50]. Von besonderem Interesse ist hierbei, dass die Akkumulation von amöboiden Mikrogliaaggregaten bzw. rekrutierten Makrophagen möglicherweise als hyperreflektive Foci in der optischen Kohärenztomographie (OCT) dargestellt werden kann [2]. OCT hat die Methode die Diagnoseund Therapieüberwachung von Netzhauterkrankungen revolutioniert und es wäre ein erheblicher Fortschritt, wenn

medgen $2017 \cdot 29: 225-233$ DOI 10.1007/s11825-017-0132-8

(c) Der/die Autor(en) 2017. Dieser Artikel ist eine Open-Access-Publikation.

\section{Karlstetter $\cdot$ K. Dannhausen $\cdot$ T. Langmann}

\section{Mikroglia und Immuntherapien bei degenerativen Netzhauterkrankungen}

\section{Zusammenfassung}

Bei allen bisher im Detail untersuchten erblichen Netzhautdegenerationen liegt eine dem Erkrankungsverlauf abträgliche chronische Aktivierung des angeborenen Immunsystems zugrunde. Vor allem residente Mikrogliazellen der Netzhaut und verschiedene Proteine des löslichen Komplementsystems tragen zu einer Schädigung von Photorezeptoren und retinalem Pigmentepithel bei. Sowohl spezifische Zielstrukturen auf reaktiven Immunzellen als auch fehlregulierte lösliche Immunmodulatoren bieten neue Ansatzpunkte für Therapien, um das Überleben der Netzhaut trotz genetischer Prädisposition zur Degeneration zu fördern. Dieser Beitrag gibt Einblick in die wesentlichen Regulationsmechanismen der Netzhautimmunologie, diskutiert die mögliche Verwendung immunologischer Biomarker für die Netzhautdiagnostik und zeigt immunmodulierende Therapieansätze durch Biologika und endogene Botenstoffe auf.

Schlüsselwörter

Netzhaut · Mikroglia $\cdot$ Komplement $\cdot$ Retinitis pigmentosa. AMD

\section{Microglia and immunomodulatory therapies for retinal degenerative diseases}

\section{Abstract}

A chronic activation of the congenital immune system that is detrimental to the course of the disease underlies all forms of hereditary retinal degeneration investigated in detail so far. Above all, resident microglial cells of the retina and various proteins of the soluble complement system contribute to damage to photoreceptors and the retinal pigment epithelium. Specific target structures on reactive immune cells in addition to dysregulated soluble immune regulators offer a new starting point for treatments to promote the survival of the retina, despite a genetic predisposition to degeneration. In this article, we provide an insight into the essential regulatory mechanisms of retinal immunology, discuss the potential use of immunological biomarkers for diagnosing retinal conditions, and demonstrate immunomodulatory therapies through the use of biological agents and endogenous messengers.

\section{Keywords}

Retina $\cdot$ Microglia $\cdot$ Complement $\cdot$ Retinitis pigmentosa. AMD damit das Niveau der Immunzellenaktivierung innerhalb der Netzhaut in vivo bestimmt werden könnte.

\section{Mikroglia bei Mausmodellen der AMD}

In Ermangelung einer echten Makula bei Nagetieren sind die meisten AMDMausmodelle auf die Untersuchung von einzelnen Merkmalen der menschlichen AMD-Pathologie beschränkt. Dazu zählen die generelle Netzhautdegeneration, die RPE-Schädigung, die Neovaskularisierung und nicht zuletzt die Immunzellenaktivierung. Dennoch sind diese Modelle sehr wertvoll, um Krankheitsmechanismen zu untersuchen und therapeutische Strategien für die AMD zu erproben.

Ein Modell für die geographische Atrophie wird durch die Immunisierung von Mäusen mit Carboxyethylpyrrol (CEP)-konjugiertem Albumin induziert, einem Peroxidationsaddukt, das in Drusen und Serum von AMD-Patienten angereichert ist [29]. Die „CEPMaus" ist bisher das einzige Modell, das in der RPE-Schicht drusenartige Ablagerungen entwickelt. Bei diesen Tieren werden proinflammatorische Mikroglia im Bereich zwischen den RPE- und Photorezeptoraußensegmenten gefunden. Bestrahlung von Mausaugen mit intensivem Weißlicht oder fokussiertem Blaulicht sind ebenfalls etablierte 


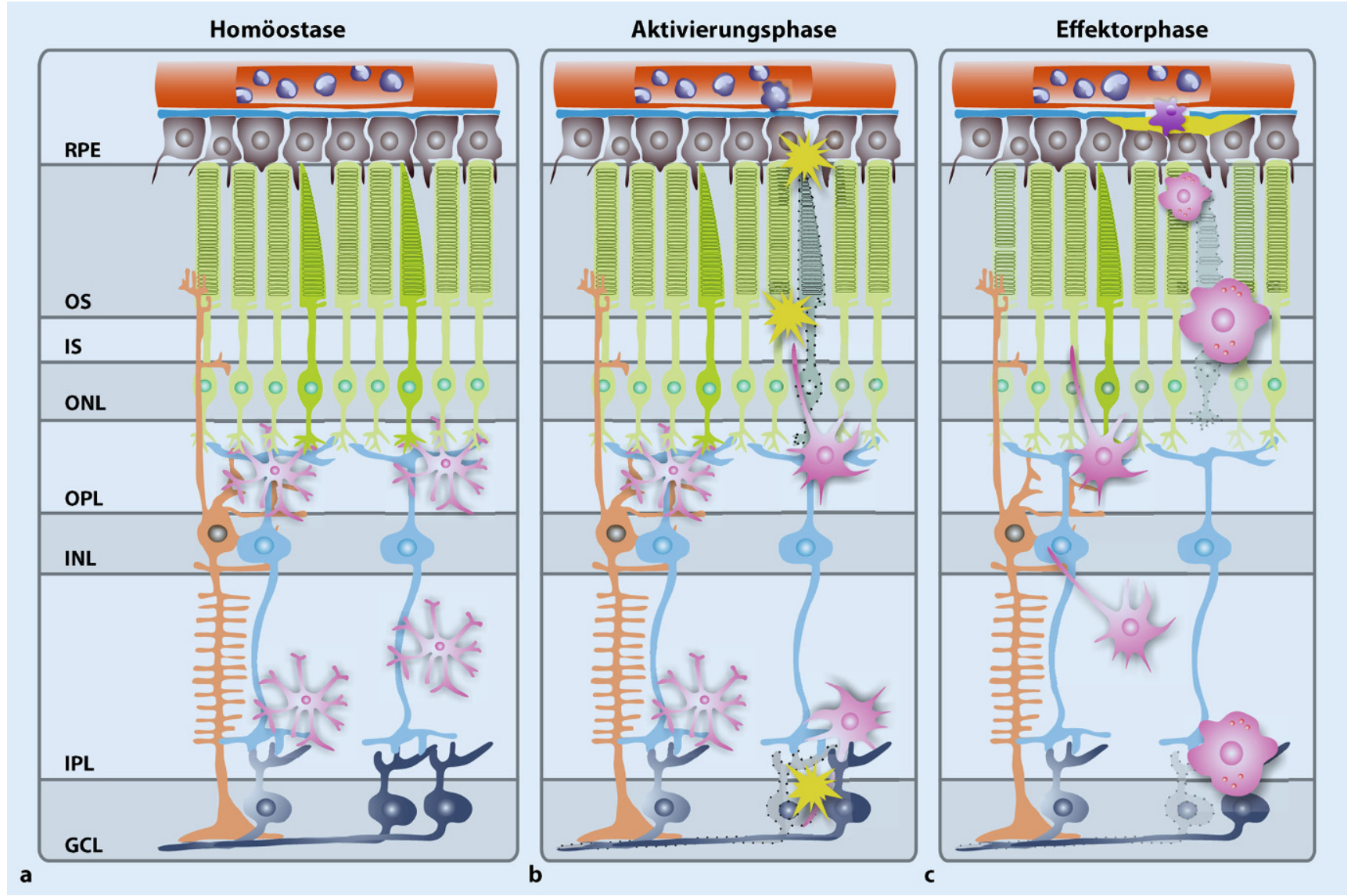

Abb. $2 \Delta$ Schaubild zu den Phasen der Mikrogliaaktivierung bei Netzhautdegenerationen. a Unter homöostatischen Bedingungen scannen Mikrogliazellen die komplette Netzhaut, wobei ihre Zellkörper in den plexiformen Schichten verbleiben und nur die motilen Fortsätze hohe Aktivität zeigen. $\boldsymbol{b}$ In der Aktivierungsphase werden Mikrogliazellen von beginnenden Degenerationsprozessen der Photorezeptorschicht (z. B. bei Retinitis pigmentosa) oder der Ganglienzellschicht (z. B. beim Glaukom) alarmiert und antworten mit einer morphologischen Transition zu amöboiden Phagozyten und Migration. c In der späteren Effektorphase sezernieren amöboide Mikrogliazellen neurotoxische Substanzen und rekrutieren durch lösliche Faktoren inflammatorische Blutzellen. (RPE retinal pigment epithelium, OS outer segments, IS inner segments, ONL outer nuclear layer, OPL outer plexiform layer, INL inner nuclear layer, IPL inner plexiform layer, GCL ganglion cell layer). (Aus [34], mit freundl. Genehmigung von Elsevier)

Modelle der trockenen AMD, die photooxidative Schäden und Netzhautdegeneration nachahmen [23]. In beiden Lichtschadensmodellen tritt mit dem Photorezeptorverlust eine starke Reaktivität von Mikrogliazellen auf, deren Rhodopsin-positive Einschlüsse den Beobachtungen bei menschlichen AMDGewebeproben ähneln [61, 62]. Die chemotaktische Antwort von Mikroglia selbst wird hauptsächlich durch photooxidativen Stress und gleichzeitige CCL2-Freisetzung aus gliotischen Müller-Zellen ausgelöst [63].

Im Gegensatz zu den degenerativen Effekten bei Lichtschadensmodellen induziert die Laser-Photokoagulation der Netzhaut einen lokalen Schaden der Bruch-Membran mit nachfolgen- der entzündungsgetriebener Neovaskularisation der Aderhaut [40, 43, 44]. Innerhalb weniger Minuten migrieren residente Mikrogliazellen zur Laserläsion und erhöhte VEGF-Spiegel fördern diesen Prozess. Es ist unwahrscheinlich, dass Mikrogliazellen selbst einen signifikanten Beitrag zur Gesamt-VEGFProduktion leisten, aber ihr Tropismus zu neu gebildeten Gefäßen könnte die Entstehung der choroidalen Neovaskularisierung (CNV) frühzeitig fördern. Bei rekrutierten CCR2+ Monozyten/ Makrophagen hingegen ist der Beitrag zur frühen VEGF-Produktion im Lasermodell der CNV gut dokumentiert [39].

\section{Mikroglia und Komplement- faktoren}

Seit Veröffentlichung der ersten genomweiten Assoziationsstudien der AMD hat das Komplementsystem beträchtliche Aufmerksamkeit im Bereich der Netzhautforschung erlangt [19]. Eine lokale Fehlregulation der alternativen Komplementfaktorkaskade in der Netzhaut gilt seither als gesicherter pathophysiologisch relevanter Mechanismus [60]. Mikroglia sind die einzigen residenten Zellen der Netzhaut, die den Komplementfaktor 3 Rezeptor exprimieren, und damit eine erhöhte Phagozytose von opsonisierten Partikeln anschalten. Mikroglia selbst sind auch in der Lage mehrere Faktoren der klassischen 

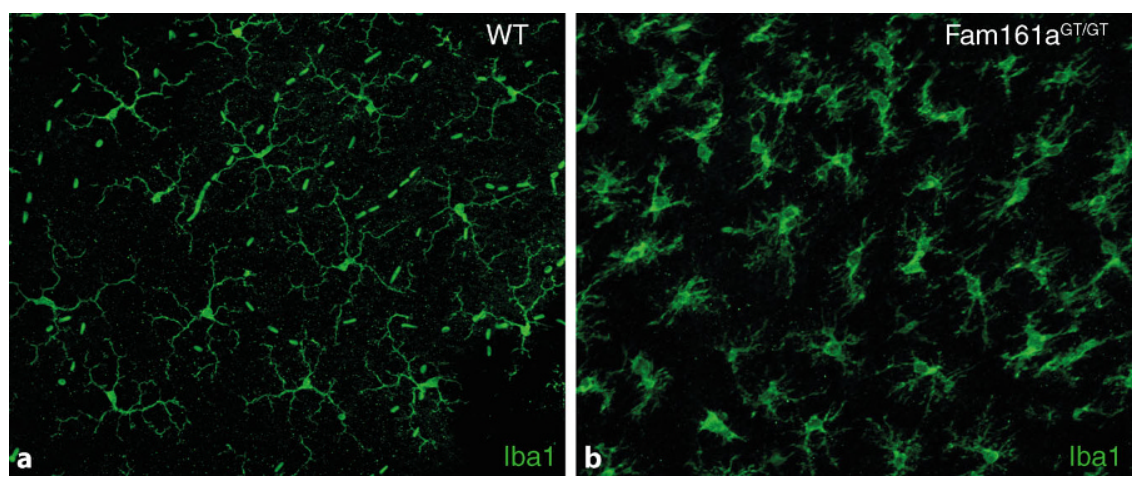

Abb. $3 \Delta$ Iba1-Immunfärbung (grün) von Mikrogliazellen in Flachpräparaten einer a wildtypischen (WT) bzw. b Fam161a-defizienten Mausnetzhaut. a In der gesunden Netzhaut bilden stark verzweigte Mikrogliazellen ein Netzwerk. b Bei retinaler Degeneration ziehen Mikrogliazellen ihre Fortsätze ein und transformieren zu amöboiden Phagozyten mit hoher migratorischer Aktivität

und alternativen Komplementkaskade zu produzieren. Besonders A2E, ein Bisretinoid des bei der AMD abgelagerten Lipofuszins, regt Mikroglia zur Produktion von C3-Aktivierungsprodukten an, die sich wiederum auf der Oberfläche von RPE-Zellen anlagern können [45].

\section{Mikroglia als Zielzelle zur therapeutischen Immunmodulation in der Netzhaut}

Reaktive Mikrogliazellen und Immunaktivierung sind übergreifende Pathomechanismen eines breiten Spektrums von Netzhauterkrankungen. Im Gegensatz $\mathrm{zu}$ individualisierten und sehr entwicklungsintensiven Gentherapieansätzen bei seltenen Netzhauterkrankungen kann durch die Modulation der Mikrogliareaktivität der Verlauf einer Vielzahl von Netzhauterkrankungen positiv beeinflusst werden. Hierbei ist es sinnvoll, die Immunaktivierung schon möglichst in Frühphasen der Erkrankung zu beeinflussen.

\section{Minozyklin}

Minozyklin ist ein halbsynthetisches Tetrazyklinderivat, das als Antibiotikum und entzündungshemmendes Medikament zur Behandlung von Akne und Arthritis eingesetzt wird. In den letzten Jahren wurde berichtet, dass Minozyklin auch bei experimentellen Modellen von verschiedenen neurodegenerativen Erkrankungen, einschließlich Parkinson,
Alzheimer, amyotropher Lateralsklerose, Multiple Sklerose und Rückenmarkverletzungen neuroprotektive Effekte ausüben kann [20]. Als Wirkmechanismus werden dabei antioxidative Effekte und die Stabilisierung von Mitochondrien diskutiert, um den Zelltod zu verhindern [55]. Am stärksten scheint jedoch die immundämpfende Wirkung auf Mikroglia, d. h. auf ihre Aktivierung, Proliferation und Migration sowie auf die Freisetzung von proinflammatorischen Zytokinen zum Tragen zu kommen [7]. Minozyklin zeigt durchweg gute Penetrationseigenschaften durch die BlutHirn- und die Blut-Retina-Schranke und hat ein positives Sicherheitsprofil [15].

Die Wirkung der Minozyklinbehandlung auf die Mikrogliaaktivierung und den Verlauf der Netzhautdegeneration wurde in verschiedenen Tiermodellen untersucht. Zhang et al. konnten im Lichtschadensmodell zeigen, dass die systemische Minozyklinbehandlung die Netzhautfunktion über eine Woche nach Lichtschaden signifikant erhalten hat [67]. Einhergehend mit dieser Beobachtung wurde auch die Anzahl an aktivierten Mikrogliazellen in der äußeren Netzhaut stark verringert [67]. Präklinische Studien aus unserer Arbeitsgruppe zur Wirkung von Minozyklin in der Netzhaut haben potente neuroprotektive Effekte gezeigt, die mit einer fast vollständigen Abwesenheit von TSPOpositiven, aktivierten Mikrogliazellen nach Lichtschaden einhergingen [62]. Darüber hinaus gibt es profunde Hinweise darauf, dass Minozyklin bei der
Modulation der Mikrogliareaktivität bei Mausmodellen erblicher Netzhautdegenerationen wirksam ist. In diesem Zusammenhang wurde gezeigt, dass die Minozyklin vermittelte Unterdrückung der Mikrogliaaktivierung direkt neuroprotektiv wirkt und zur Erhaltung der Netzhautstruktur und -funktion in Mertk-knockout und rd10-Mäusen beiträgt $[38,54]$.

Basierend auf vielversprechenden präklinischen Daten für Minozyklin wurden am National Institutes of Health (NIH) klinische Studien für das diabetische Makulaödem (NTC01120899) und den retinalen Venenverschluss initiiert (NTC01468844). Bei Patienten mit diabetischer Retinopathie wurde über die Dauer von sechs Monaten zweimal täglich $100 \mathrm{mg}$ Minozyklin oral verabreicht. Im Studienverlauf verbesserten sich die visuelle Funktion und das zentrale Makulaödem und die vaskuläre Leckage war bei allen Studienteilnehmern zurückgebildet [12]. Es konnte ausgeschlossen werden, dass diese Effekte mit Veränderungen von diabetischen Labormarkern wie dem glykämischen Index oder der Serumkreatinin assoziiert waren, vielmehr wurden sie durch die Verminderung von lokalen entzündlichen Effekten vermittelt. Weitere Erkenntnisse in diesem Bereich bedürfen jedoch umfassender Phase-II-Studien mit einem PlaceboArm und dem Vergleich mit etablierten Anti-VEGF-Therapien.

Minozyklin wurde auch in einer experimentellen Behandlung eines Patienten mit Retinitis pigmentosa angewendet. In diesem 140-monatigen Heilungsversuch wurden täglich $100 \mathrm{mg}$ Minozyklin zusammen mit der neuroprotektiven Substanz Deprenyl verabreicht [5]. Die progrediente Einschränkung des Gesichtsfeldes verlief in Anwesenheit der Behandlung erheblich langsamer als in der früheren Krankheitsphase vor der Medikation. Auch hier müssen Studien an größeren Kohorten mit Minozyklin alleine durchgeführt werden, um das Behandlungsschema zu optimieren und die Wirkung der Behandlung auf das Fortschreiten der Retinitis pigmentosa systematisch zu erfassen. 


\section{Docosahexaensäure (DHA)}

Die mehrfach ungesättigte Fettsäure DHA ist in Phospholipiden der Plasmamembran von Photorezeptoren stark angereichert und ist gleichzeitig ein Vorläufer von Neuroprotektin D1, einem Faktor, der das Überleben von Photorezeptoren und RPE-Zellen fördert [6]. Darüber hinaus wirkt DHA starkimmunmodulierend und trägt zur Hemmung der entzündlichen Aktivierung von $\mathrm{Mi}$ kroglia bei $[3,18]$. Da die DHA-Spiegel in verschiedenen menschlichen erblichen Formen sowie Tiermodellen der Netzhautdegeneration vermindert sind, werden diese Fettsäuren als therapeutische Nahrungsergänzungsmittel angesehen. Es gab zunächst widersprüchliche Ergebnisse der Wirkung von DHA bei der Verzögerung der Netzhautdegeneration. So konnte die Fischölsupplementierung bei Hunden mit einer progressiven Stäbchen-Zapfen-Dystrophie oder bei transgenen Ratten mit einem Retinitispigmentosa-Phänotyp das Fortschreiten der Degeneration nicht beeinflussen [1, 47]. Im Gegensatz dazu verzögerte die Nahrungsergänzung mit DHA bei Mausmodellen der X-gebundenen Retinoschisis die Netzhautdegeneration signifikant [18] und hemmte die Mikrogliaaktivierung in Modellen der neuronalen Ceroid-Lipofuszinose [48] und im transgenen ELOVL4 StargardtMausmodell [16].

Eine vierjährige placebokontrollierte Studie mit 51 Patienten mit X-chromosomaler Retinitis pigmentosa ergab, dass eine hochdosierte DHA-Supplementierung ein sehr gutes Sicherheitsprofil zeigte und die Sensitivität des Gesichtsfelds signifikant erhalten konnte [28]. Der Erhalt des Gesichtsfelds war dabei direkt mit der Normalisierung des DHASpiegels im Blut korreliert. Ähnlich dazu korrelieren hohe Plasma-DHA-Konzentrationen nach Nahrungsergänzung mit dem Erhalt der Netzhautfunktion und Sehschärfe bei Kindern mit langkettiger 3-Hydroxyacyl-CoA-Dehydrogenasedefizienz [22]. Das Timing und die Dosis der DHA-Supplementierung sind sehr wahrscheinlich entscheidend, um die Wirksamkeit einer DHA-Supplementation zu optimieren. Dementsprechend haben die meisten Studien, die positive Effekte durch DHA bei der Netzhautdegeneration zeigen konnten, die Behandlung relativ frühzeitig begonnen.

\section{Präklinische immun- modulatorische Ansätze}

Die präklinische Forschung der letzten Jahre folgte dem übergeordneten Konzept der Auswahl solcher Substanzen, die sowohl auf die zelluläre Immunaktivierung als auch auf die Mikroumgebung der Netzhaut neuroprotektiv wirken (siehe Minozyklin und DHA). Als Paradebeispiel sind hier natürliche Komponenten der extrazellulären Matrix zu nennen. Endogene Zuckerstrukturen wie ein von Chondroitinsulfat-Proteoglykanen abgeleitetes Disaccharid (CSPG-DS) schützen lokal vor dem Verlust an Netzhautneuronen in experimentellen Mausmodellen des Glaukoms und der autoimmunen Uveitis [4, 58]. Dabei induziert CSPG-DS einen regulatorischen Mikrogliaphänotyp mit erhöhter phagozytischer Kapazität, aber ohne die gleichzeitige Produktion von neurotoxischen Sauerstoffradikalen [17]. Andere von der extrazellulären Matrix abgeleitete Moleküle mit einer spezifischen und wirksamen Mikroglia inhibierenden Wirkung sind Sialinsäurereste an Glykoproteinen. Jüngste Arbeiten aus unserer Arbeitsgruppe konnten zeigen, dass die intravitreale Injektion von niedermolekularen Polysialinsäuren die Mikrogliareaktivität nach laserinduziertem Netzhautschaden vermindert sowie die Komplementaktivierung in vitro und in vivo potent unterdrückt [35]. Die synergistische Wirkung der Polysialinsäuren auf Mikroglia-Siglec-Rezeptoren einerseits und auf das alternative Komplementsystem andererseits greift damit erstmals sowohl beim zellulären als auch beim löslichen angeborenen Immunsystem ein.

Als weitere therapeutische Zielstruktur ist das mitochondriale Translokatorprotein $(18 \mathrm{kDa})$ (TSPO) zu nennen, das in aktivierten murinen und humanen Netzhaut-Mikrogliazellen überexprimiert wird [32]. In weiterführenden Studien konnte gezeigt werden, dass der selektive TSPO-Ligand XBD173 die Anzahl amöboider Mikroglia in degenerierenden retinalen Explantaten effektiv reduziert und darüber hinaus neuroprotektiv in verschiedenen Lichtschadensmodellen der Maus wirkt [61]. Da TSPO-Liganden auch als Therapieoption für neurologische Erkrankungen gelten, erscheint eine gemeinsame Weiterentwicklung auch für degenerative Netzhauterkrankungen sinnvoll.

Der sekundäre Pflanzenstoff Curcumin ist eine weitere vielversprechende Verbindung, die auf mikrogliale Zielstrukturen wirkt und gleichzeitig das neuronale Überleben positiv beeinflusst. Curcumin blockiert die Produktion von Sauerstoffradikalen, reduziert die Sekretion von entzündungshemmenden Zytokinen und hemmt die Migration von Mikroglia in vitro [31]. Es gibt bereits Hinweise, dass Curcumin protektive Effekte in Tiermodellen hat. Hier wurde gezeigt, dass es gegen die Mikroglia vermittelte Neurotoxizität bei akutem Lichtschaden schützt, die retinale Degeneration bei neuronaler CeroidLipofuszinose verzögert, das Überleben von Photorezeptoren bei transgenen Ratten mit der P23H-Rhodopsin-Mutation verbessert und die experimentelle diabetische Retinopathie bei Ratten vermindert [26, 46, 48, 64]. Vor allem auf dem Gebiet der Formulierungsentwicklung besteht noch Entwicklungsbedarf, um optimale Anwendungsformen für Curcumin zu finden, um seine orale Bioverfügbarkeit zu verbessern.

\section{Ausblick}

Es gibt überzeugende Daten dafür, dass die Mikrogliareaktivität ein frühes und übergreifendes Kennzeichen bei degenerativen Netzhauterkrankungen mit verschiedenen zugrunde liegenden Krankheitsursachen ist. In vitro-Experimente und Tiermodelle waren enorm hilfreich, um die wichtigsten Wirkmechanismen der Mikrogliareaktivität bei Netzhautverletzungen zu charakterisieren. Neben ihrer Rolle als frühe Biomarker des Gewebsschadens sind Mikroglia aktive Bestandteile des Netzhautimmunsystems, die chronische Entzündungsreaktionen auslösen können. Die Untersuchung der zellulären Immunaktivierung in Tiermodellen für seltene und multifaktorielle 
Netzhautdegenerationen hat bereits neue Erkenntnisse für immunmodulatorische, mikrogliagerichtete Therapieansätze hervorgebracht.

In Zukunft wird es von größter Bedeutung sein, sich auf Studien mit menschlichem Netzhautgewebe zu fokussieren. Spenderbiobanken mit Netzhautgewebe und entsprechende DNA- und Blutproben können extrem hilfreich sein, um Gemeinsamkeiten und Unterschiede in der retinalen Immunhomöostase bei Tiermodellen und im Menschen $\mathrm{zu}$ verstehen. Es wird auch eine große Herausforderung sein, mikrogliagerichtete Therapieoptionen in translationalen bzw. klinischen Studien zu testen, um die vielversprechendsten Ansätze auszuwählen. Da immunmodulierende Ansätze in Verbindung mit einer neuroprotektiven Behandlung am effektivsten zu sein scheinen, ist es sehr wahrscheinlich, dass ein kombinierter Ansatz erforderlich ist. Ein grundlegendes Problem bei klinischen Studien zur Behandlung von Entzündungserkrankungen der Netzhaut und Immuntherapien ist der derzeitige Mangel an geeigneten Biomarkern zur Endpunktbestimmung. Es gibt gute Hinweise, dass sich die Dysregulation des Immunsystems teilweise bei Patienten in systemischen Parametern widerspiegelt. Letztlich wird es dennoch wichtig sein, sich auf das okuläre Immunsystem $\mathrm{zu}$ konzentrieren, um lokale entzündliche Prozesse durch die Analyse von Zytokinen und Immunzellenpopulationen beispielsweise im Kammerwasser von Patienten zu bestimmen. Es besteht auch eine gute Chance, dass die Immunzellreaktivität in der menschlichen Netzhaut durch verbesserte in vivo Imaging-Technologien nachgewiesen werden kann. Mit dieser spannenden Perspektive kann die Netzhautforschung neuartige Konzepte für mikrogliazielgerichtete Therapieansätze entwickeln - eine lohnende Aufgabe im Sinne der vielen Patienten mit Netzhautdystrophien.

\section{Korrespondenzadresse}

Prof. Dr. rer. nat. T. Langmann

Lehrstuhl für Experimentelle Immunologie des Auges, Zentrum für Augenheilkunde, Uniklinik Köln

Joseph-Stelzmann-Str. 9, 50931 Köln,

Deutschland

thomas.langmann@uk-koeln.de

\section{Einhaltung ethischer Richtlinien}

Interessenkonflikt. T. Langmann ist Mitglied der Expertenkommission des Förderprogramms für Augenheilkunde von Bayer und erhielt Beraterhonorare der Firmen Bayer, Novartis und Roche im Bereich der experimentellen Ophthalmologie. M. Karlstetter und K. Dannhausen geben an, dass kein Interessenkonflikt besteht.

Alle beschriebenen Untersuchungen am Menschen wurden mit Zustimmung der zuständigen Ethik-Kommission, im Einklang mit nationalem Recht sowie gemäß der Deklaration von Helsinki von 1975 (in der aktuellen, überarbeiteten Fassung) durchgeführt. Von allen beteiligten Patienten liegt eine Einverständniserklärung vor. Alle nationalen Richtlinien zur Haltung und zum Umgang mit Labortieren wurden eingehalten und die notwendigen Zustimmungen der zuständigen Behörden liegen vor.

Open Access. Dieser Artikel wird unter der Creative Commons Namensnennung 4.0 International Lizenz (http://creativecommons.org/licenses/by/4.0/deed. de) veröffentlicht, welche die Nutzung, Vervielfältigung, Bearbeitung, Verbreitung und Wiedergabe in jeglichem Medium und Format erlaubt, sofern Sie den/die ursprünglichen Autor(en) und die Quelle ordnungsgemäßnennen, einen Linkzur Creative Commons Lizenz beifügen und angeben, ob Änderungen vorgenommen wurden.

\section{Literatur}

1. Aguirre GD, Acland GM, Maude MB et al (1997) Diets enriched in docosahexaenoic acid fail to correct progressive rod-cone degeneration (prcd) phenotype. Invest Ophthalmol Vis Sci 38:2387-2407

2. Altay L, Scholz P, Schick T et al (2016) Association of hyperreflective foci present in early forms of agerelated macular degeneration with known agerelated macular degeneration risk polymorphisms. Invest Ophthalmol Vis Sci 57:4315-4320

3. Antonietta Ajmone-Cat M, Lavinia Salvatori $M$, De Simone $\mathrm{R}$ et al (2012) Docosahexaenoic acid modulates inflammatory and antineurogenic functions of activated microglial cells. J Neurosci Res 90:575-587

4. Bakalash S, Rolls A, Lider O et al (2007) Chondroitin sulfate-derived disaccharide protects retinal cells from elevated intraocular pressure in aged and immunocompromised rats. Invest Ophthalmol Vis Sci 48:1181-1190

5. Baumgartner WA, Baumgartner AM (2013) Rationale for an experimental treatment of retinitis pigmentosa: 140-month test of hypothesis with one patient. Med Hypotheses 81:720-728
6. Bazan NG, Calandria JM, Serhan CN (2010) Rescue and repair during photoreceptor cell renewal mediated by docosahexaenoic acid-derived neuroprotectin D1. J Lipid Res 51:2018-2031

7. Beattie MS (2004) Inflammation and apoptosis: linked therapeutic targets in spinal cord injury. Trends Mol Med 10:580-583

8. Berger W, Kloeckener-Gruissem B, Neidhardt J (2010) The molecular basis of human retinal and vitreoretinal diseases. Prog Retin Eye Res 29:335-375

9. Bialas AR, Stevens B (2013) TGF-beta signaling regulates neuronal $\mathrm{C} 1 \mathrm{q}$ expression and developmental synaptic refinement. Nat Neurosci 16:1773-1782

10. Cardona AE, Pioro EP, Sasse ME et al (2006) Control of microglial neurotoxicity by the fractalkine receptor. Nat Neurosci 9:917-924

11. Chang B, Hurd R, Wang J et al (2013) Survey of common eye diseases in laboratory mouse strains. Invest Ophthalmol Vis Sci 54:4974-4981

12. Cukras CA, Petrou P, Chew EY et al (2012) Oral minocycline for the treatment of diabetic macular edema (DME): results of a phase I/II clinical study. Invest Ophthalmol Vis Sci 53:3865-3874

13. Damani MR, Zhao L, Fontainhas AM et al (2011) Age-related alterations in the dynamic behavior of microglia. Aging Cell 10:263-276

14. Diaz-Araya CM, Provis JM, Penfold PL et al (1995) Development of microglial topography in human retina. JComp Neurol 363:53-68

15. Domercq M, Matute C (2004) Neuroprotection by tetracyclines. Trends Pharmacol Sci 25:609-612

16. Dornstauder B, Suh M, Kuny S et al (2012) Dietary docosahexaenoic acid supplementation prevents age-related functional losses and A2E accumulation in the retina. Invest Ophthalmol Vis Sci 53:2256-2265

17. Ebert S, Schoeberl T, Walczak Y et al (2008) Chondroitin sulfate disaccharide stimulates microglia to adopt a novel regulatory phenotype. JLeukocBiol 84:736-740

18. Ebert S, Weigelt K, Walczak Y et al (2009) Docosahexaenoic acid attenuates microglial activation and delays early retinal degeneration. JNeurochem 110:1863-1875

19. Fritsche LG, Fariss RN, Stambolian D et al (2014) Age-related macular degeneration: genetics and biology coming together. Annu Rev Genomics Hum Genet 15(1):151-171. doi:10.1146/annurevgenom-090413-025610

20. Garrido-Mesa N, Zarzuelo A, Galvez J (2013) Minocycline: far beyond an antibiotic. $\mathrm{Br} J$ Pharmacol 169:337-352

21. Gehrig A, Langmann T, Horling F et al (2007) Genome-wide expression profiling of the retinoschisin-deficient retina in early postnatal mouse development. Invest Ophthalmol Vis Sci 48:891-900

22. Gillingham MB, Weleber RG, Neuringer $M$ et al (2005) Effect of optimal dietary therapy upon visual function in children with long-chain 3-hydroxyacyl $\mathrm{CoA}$ dehydrogenase and trifunctional protein deficiency. Mol Genet Metab 86:124-133

23. Grimm C, Reme CE (2013) Light damage as a model of retinal degeneration. Methods Mol Biol 935:87-97

24. Guo C, Otani A, Oishi A et al (2012) Knockout of ccr2 alleviates photoreceptor cell death in a model of retinitis pigmentosa. Exp Eye Res 104:39-47

25. Gupta N, Brown KE, Milam AH (2003) Activated microglia in human retinitis pigmentosa, lateonset retinal degeneration, and age-related macular degeneration. Exp Eye Res 76:463-471 
26. Gupta SK, Kumar B, Nag TC et al (2011) Curcumin prevents experimental diabetic retinopathy in rats through its hypoglycemic, antioxidant, and antiinflammatory mechanisms. JOcul Pharmacol Ther 27:123-130

27. Hoek RM, Ruuls SR, Murphy CA et al (2000) Down-regulation of the macrophage lineage through interaction with OX2 (CD200). Science 290:1768-1771

28. Hoffman DR, Hughbanks-Wheaton DK, Spencer $R$ et al (2015) Docosahexaenoic acid slows visual field progression in $\mathrm{X}$-linked retinitis pigmentosa: ancillary outcomes of the DHAX trial. Invest Ophthalmol Vis Sci 56:6646-6653

29. Hollyfield JG, Bonilha VL, Rayborn ME et al (2008) Oxidative damage-induced inflammation initiates age-related macular degeneration. Nat Med 14:194-198

30. Jager RD, Mieler WF, Miller JW (2008) Agerelated macular degeneration. $\mathrm{N}$ Engl J Med 358:2606-2617

31. Karlstetter M, Lippe E, Walczak Y et al (2011) Curcumin is a potent modulator of microglial gene expression and migration. J Neuroinflammation 8:125

32. Karlstetter M, Nothdurfter C, Aslanidis A et al (2014) Translocator protein (18kDa) (TSPO) is expressed in reactive retinal microglia and modulates microglial inflammation and phagocytosis. JNeuroinflammation 11:3

33. Karlstetter M, Sorusch N, Caramoy A et al (2014) Disruption of the retinitis pigmentosa 28 gene Fam161a in mice affects photoreceptor ciliary structure and leads to progressive retinal degeneration. Hum Mol Genet 23(19):5197-5210. doi: $10.1093 / \mathrm{hmg} / \mathrm{ddu} 242$

34. Karlstetter M, Scholz R, Rutar M et al (2015) Retinal microglia: just bystander or target for therapy? Prog Retin Eye Res 45:30-57

35. Karlstetter M, Kopatz J, Aslanidis A et al (2017) Polysialic acid blocks mononuclear phagocyte reactivity, inhibits complement activation, and protects from vascular damage in the retina. EMBO Mol Med 9:154-166

36. Keeler CE (1924) The inheritance of a retinal abnormality in white mice. Proc Natl Acad Sci U SA 10:329-333

37. Killingsworth MC, Sarks JP, Sarks SH (1990) Macrophages related to Bruch's membrane in age-related macular degeneration. Eye (Lond) 4(4):613-621

38. Kohno H, Chen Y, Kevany BM et al (2013) Photoreceptor proteins initiate microglial activation via Toll-like receptor 4 in retinal degeneration mediated by all-trans-retinal. J Biol Chem 288:15326-15341

39. Krause TA, Alex AF, Engel DR et al (2014) VEGFproduction by CCR2-dependent macrophages contributes to laser-induced choroidal neovascularization. PLOSONE 9:e94313

40. Lambert V, Lecomte J, Hansen S et al (2013) Laserinduced choroidal neovascularization model to study age-related macular degeneration in mice. Nat Protoc 8:2197-2211

41. Langmann $T$ (2007) Microglia activation in retinal degeneration. JLeukoc Biol 81:1345-1351

42. Langmann T, Di Gioia SA, Rau I et al (2010) Nonsense mutations in FAM161A cause RP28associated recessive retinitis pigmentosa. Am J Hum Genet 87:376-381

43. Luckoff A, Caramoy A, Scholz R et al (2016) Interferon-beta signaling in retinal mononuclear phagocytes attenuates pathological neovascularization. EMBO Mol Med 8:670-678
44. Luckoff A, Scholz R, Sennlaub F et al (2017) Comprehensive analysis of mouse retinal mononuclear phagocytes. Nat Protoc 12:1136-1150

45. Ma W, Coon S, Zhao L et al (2013) A2E accumulation influences retinal microglial activation and complement regulation. Neurobiol Aging 34:943-960

46. Mandal MN, Patlolla JM, Zheng L et al (2009) Curcumin protects retinal cells from light-and oxidant stress-induced cell death. Free Radic Biol Med 46:672-679

47. Martin RE, Ranchon-Cole I, Brush RS et al (2004) P23H and S334ter opsin mutations: Increasing photoreceptor outer segment n-3 fatty acid content does not affect the course of retinal degeneration. Mol Vis 10:199-207

48. Mirza M, Volz C, Karlstetter M et al (2013) Progressive retinal degeneration and glial activation in the CLN6 (nclf) mouse model of neuronal ceroid lipofuscinosis: a beneficial effect of DHA and curcumin supplementation. PLOSONE 8:e75963

49. Nathan C, Muller WA (2001) Putting the brakes on innate immunity: a regulatory role for CD200? Nat Immunol 2:17-19

50. Newman AM, Gallo NB, Hancox LS et al (2012) Systems-level analysis of age-related macular degeneration reveals global biomarkers and phenotype-specific functional networks. Genome Med 4:16

51. Paglinawan R, Malipiero U, Schlapbach R et al (2003) TGFbeta directs gene expression of activated microglia to an anti-inflammatory phenotype strongly focusing on chemokine genes and cell migratory genes. Glia 44:219-231

52. Paolicelli RC, Bolasco G, Pagani F et al (2011) Synaptic pruning by microglia is necessary for normal brain development. Science 333:1456-1458

53. Penfold PL, Madigan MC, Gillies MC et al (2001) Immunological and aetiological aspects of macular degeneration. Prog Retin Eye Res 20:385-414

54. Peng B, Xiao J, Wang K et al (2014) Suppression of microglial activation is neuroprotective in a mouse model of human retinitis pigmentosa. J Neurosci 34:8139-8150

55. Plane JM, Shen Y, Pleasure DE et al (2010) Prospects for minocycline neuroprotection. Arch Neurol 67:1442-1448

56. Provis JM (2001) Development of the primate retinal vasculature. Prog Retin Eye Res 20:799-821

57. Rohrer B, Pinto FR, Hulse KE et al (2004) Multidestructive pathways triggered in photoreceptor cell death of the rd mouse as determined through gene expression profiling. JBiol Chem 279:41903-41910

58. Rolls A, Cahalon L, Bakalash S et al (2006) A sulfated disaccharide derived from chondroitin sulfate proteoglycan protects against inflammation-associated neurodegeneration. FASEB J 20:547-549

59. Schafer DP, Lehrman EK, Kautzman AG et al (2012) Microglia sculpt postnatal neural circuits in an activity and complement-dependent manner. Neuron 74:691-705

60. Schick T, Steinhauer M, Aslanidis A et al (2017) Local complement activation in aqueous humor in patients with age-related macular degeneration. Eye (Lond) 31(5):810-813. doi:10.1038/eye.2016 328

61. Scholz R, Caramoy A, Bhuckory MB et al (2015) Targeting translocator protein (18 kDa) (TSPO) dampens pro-inflammatory microglia reactivity in the retina and protects from degeneration. JNeuroinflammation 12:201

62. Scholz R, Sobotka M, Caramoy A et al (2015) Minocycline counter-regulates pro-inflammatory microglia responses in the retina and protects from degeneration. J Neuroinflammation 12:209

63. Suzuki M, Tsujikawa M, Itabe H et al (2012) Chronic photo-oxidative stress and subsequent MCP-1 activation as causative factors for age-related macular degeneration. JCell Sci 125:2407-2415

64. Vasireddy V, Chavali VR, Joseph VT et al (2011) Rescue of photoreceptor degeneration by curcumin in transgenic rats with $\mathrm{P} 23 \mathrm{H}$ rhodopsin mutation. PLOSONE6:e21193

65. Weber BH, Schrewe H, Molday LL et al (2002) Inactivation of the murine $\mathrm{X}$-linked juvenile retinoschisis gene, Rs $1 \mathrm{~h}$, suggests a role of retinoschisin in retinal cell layer organization and synaptic structure. Proc Natl Acad Sci U S A 99:6222-6227

66. Yoshida N, Ikeda Y, Notomi Set al (2013) Laboratory evidence of sustained chronic inflammatory reaction in retinitis pigmentosa. Ophthalmology 120:e5-e12

67. Zhang C, Lei B, Lam TTet al (2004) Neuroprotection of photoreceptors by minocycline in light-induced retinal degeneration. Invest Ophthalmol Vis Sci 45:2753-2759 
Hier steht eine Anzeige.

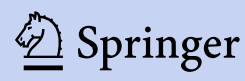

